\title{
Resonant Tunneling of Polarized Electrons Through Nonmagnetic III-V Semiconductor Multiple Barriers
}

\author{
C. Moysés Araújo, A. Ferreira da Silva, \\ Instituto de Física Universidade Federal da Bahia, \\ UFBa, Salvador, BA, Brazil \\ and E. A. de Andrada e Silva \\ Instituto Nacional de Pesquisas Espaciais - INPE, \\ 12201-900, S.J. dos Campos, SP, Brazil
}

Received on 23 April, 2001

\begin{abstract}
The quantum transport of spin-polarized electrons across nonmagnetic III-V semiconductor multiple barriers is considered theoretically. We have calculated the spin dependent transmission coefficient, for conducting electrons transversing lattice-matched $\mathrm{In}_{0.53} \mathrm{Ga}_{0.47} \mathrm{As} / \mathrm{GaAs}_{0.5} \mathrm{Sb}_{0.5} / \mathrm{In}_{0.53} \mathrm{Ga}_{0.47} \mathrm{As} /$ $\mathrm{InP} / \mathrm{In}_{0.53} \mathrm{Ga}_{0.47} \mathrm{As}$ nanostructures with different numbers of asymmetric double barriers, as a function of electron energy and angle of incidence. Spin-orbit split resonances, due to the Rashba term, are observed. The envelope function approximation and the Kane k·p model for the bulk are used. For an unpolarized incident beam of electrons, we also obtain the spin polarization of the transmitted beam. The formation of spin dependent minibands of energy with nonzero transmission is observed.
\end{abstract}

The possibility of electron-spin polarization by resonant tunneling, due to the Rashba spin-orbit coupling in semiconductor heterostructures, has been recently proposed [1, 2]. Such spin-dependent effect is of interest in the development of the so called spintronics $[3,4,5]$. The material or structure paramater optimization of such effect is however still missing. In this contribuition, we discuss the results for both spindependent transmission coefficient and polarization for electrons traversing multiple barriers with varying number of asymmetric double barrier unit cells. Spindependent minibands of energies with nozero transmission and an increasing maximum polarization of the transmitted beam, for increasing number of cells, are obtained .

It is well known that the so called Rashba spin-orbit term in the effective Hamiltonian for electrons confined in asymmetric quantum wells depends only on the angle $\theta$ between the growth direction $(\widehat{z})$ and the electron's wave-vector $\mathbf{k}$. It can be written as [6]

$$
H_{S O}=\frac{d}{d z} \beta(z, E) k \sin \theta=\frac{d}{d z} \beta(z, E) k_{\|} \cdot
$$

The coupling parameter $\beta$ as given by the eigth band
Kane model reads [7]

$$
\beta(z, E)=\frac{P^{2}}{2}\left(\frac{1}{E-E_{v}(z)}-\frac{1}{E-E_{v}(z)+\Delta(z)}\right)
$$

where $E_{v}$ is the edge of the valence band, $\Delta(z)$ is the spin orbit splitting in the maximum of the valence band and $\mathrm{P}$ is the interband momentum matrix element. Simple spin dependent boundary conditions for the envelope function can be derived in the presence of this term [7] and the problem of the spin-dependent quantum transport can be studied with standard wave mechanics procedure (note that for zero bias $H_{s o}=0$ in each layer of the structure and the solution there remains a plane wave).

We have then considered an incoming electron with energy E, wave-vector $k_{\|}$parallel to the planes and spin + or - (up or down with respect to $\widehat{y})$, and solved for the spin dependent transmission coefficient $t_{ \pm}$, for different number of asymmetrical double barrier unit cells. Using standard transfer matrix method the solution is straightforward. For the two cells case, for example, we must solve the following equation

$$
\left(\begin{array}{c}
t_{ \pm} \\
0
\end{array}\right)=M_{ \pm}^{2}\left(\begin{array}{cc}
e^{i k_{z} 2 p} & 0 \\
0 & e^{-i k_{z} 2 p}
\end{array}\right)\left(\begin{array}{c}
1 \\
r_{ \pm}
\end{array}\right)
$$


with

$$
M_{ \pm}=B_{ \pm}^{(1)}\left(\begin{array}{cc}
e^{-i k_{z} w_{1}} & 0 \\
0 & e^{i k_{z} w_{1}}
\end{array}\right) B_{ \pm}^{(2)}\left(\begin{array}{cc}
e^{-i k_{z} w_{2}} & 0 \\
0 & e^{i k_{z} w_{2}}
\end{array}\right)
$$

and

$$
w_{j}=d_{j}+L_{j}, \quad p=w_{1}+w_{2}
$$

where the $B_{ \pm}^{(j)}, j=1,2$ are the spin-dependent transfer matrices corresponding to the two different barriers, $L_{1}$ is the distance from barrier 1 to barrier 2 , while $L_{2}$ is from barrier 2 to barrier $1, d_{j}$ is the $j^{t h}$-barrier width and $\hbar k_{z}=\sqrt{2 m_{0}(E) E} \cos \theta$ is the electron's momentum along the growth direction. The transfer matrix can be obtained directly from the spin-dependent boundary conditions [7] and can be written as [1]

$$
\begin{aligned}
B_{ \pm}^{(j)}= & \frac{m_{0} m_{j}}{2 k_{z} \rho_{j}} \sinh \left(\rho_{j} d_{j}\right)\left(\begin{array}{cc}
e^{-i k_{z} d_{j}} & 0 \\
0 & e^{i k_{z} d_{j}}
\end{array}\right) \\
& \times\left(\begin{array}{cc}
P & Q_{ \pm} \\
Q_{ \pm}^{*} & P^{*}
\end{array}\right)
\end{aligned}
$$

with

$$
\begin{aligned}
P= & \frac{2 k_{z} \rho_{j}}{m_{0} m_{j}} \frac{1}{\tanh \left(\rho_{j} d_{j}\right)}+ \\
& i\left[\left(\frac{2 k_{\|}}{\hbar^{2}}\right)^{2}\left(\beta_{0}-\beta_{j}\right)^{2}+\left(\frac{k_{z}^{2}}{m_{0}^{2}}-\frac{\rho_{j}^{2}}{m_{j}^{2}}\right)\right]
\end{aligned}
$$

and

$$
\begin{aligned}
& Q_{ \pm}= \pm \frac{4 k_{z} k_{\|}}{\hbar^{2} m_{0}}\left(\beta_{0}-\beta_{j}\right)+ \\
& i\left[\left(\frac{2 k_{\|}}{\hbar^{2}}\right)^{2}\left(\beta_{0}-\beta_{j}\right)^{2}-\left(\frac{k_{z}^{2}}{m_{0}^{2}}+\frac{\rho_{j}^{2}}{m_{j}^{2}}\right)\right],
\end{aligned}
$$

where, $\hbar k_{\|}=\sqrt{2 m_{0}(E) E} \sin \theta$ is the conserved momentum parallel to the interfaces and $\rho_{j}=$ $\sqrt{2 m_{j}(E)\left(E_{c}^{j}-E\right) / \hbar^{2}+k_{\|}^{2}}$ is the decay coefficient of the evanescent wave inside the $j^{t h}$-barrier. The well and barrier material parameter, $\left\{m_{0}, \beta_{0}\right\}$ and $\left\{m_{j}, \beta_{j}\right\}$ respectively, are energy dependent, in accord to the Kane model.

In Fig. 1 we show the results of the spin dependent transmission probability $T_{ \pm}=t_{ \pm} t_{ \pm}^{*}$ as a function of electron energy in the case of a multiple double-barrier structure with three unit cells, corresponding to a structure with six barriers. The band parameters used in the calculation are listed in Table 1 . One can see that instead of the broad resonances found for one double barrier $[1,2]$, one can see in this multiple barrier system the formation of spin dependent minibands of energies with nonzero transmission. It is interesting to note that the miniband width is much larger than the spin-splitting obtained with just one unit cell. For this case with $L_{1}=L_{2}$, as we add more and more cells the structure loses gradually its inversion asymmetry and the oposite spin minibands tend to overlap completely, reestablishing the spin degeneracy of the symmetric structure.

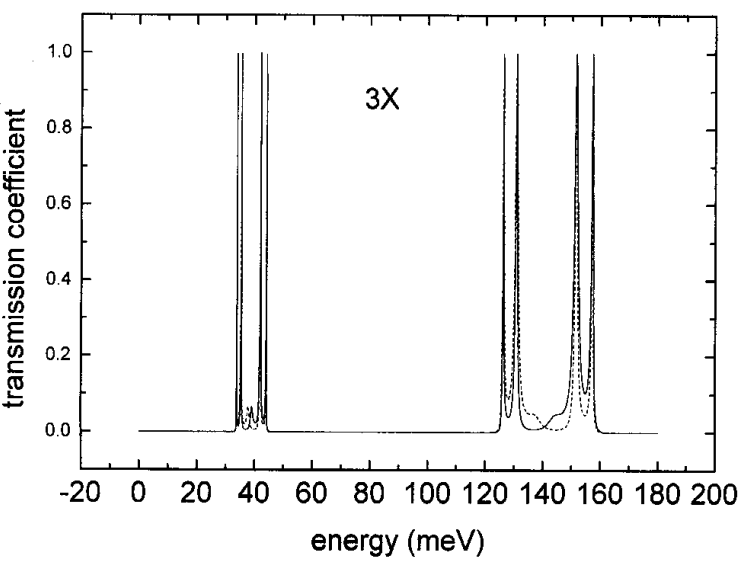

Figure 1. Transmission coefficient for electrons arriving with an angle $\theta=\pi / 4$ with respect to the growth direction, and crossing three (3X) asymmetric double-barrier unit cells of lattice-matched $\operatorname{In}_{0.53} \mathrm{Ga}_{0.47} \mathrm{As} / \mathrm{InP} / \mathrm{In} 0.53$ $\mathrm{Ga}_{0.47} \mathrm{As} / \mathrm{GaAs}_{0.5} \mathrm{Sb}_{0.5} / \mathrm{In}_{0.53} \mathrm{Ga}_{0.47} \mathrm{As}$. We have used $L_{1}=L_{2}=20 \mathrm{~nm}$ and $d_{1}=d_{2}=3 \mathrm{~nm}$ The band parameters used are listed in Table 1 and the band offsets for the conduction band were $0.18 \mathrm{eV}$ and $0.36 \mathrm{eV}$ for InGaAs/InP and InGaAs/GaAsSb respectively, which were recently measured [8].

\begin{tabular}{cccc}
\hline & $E_{g}(e V)$ & $\Delta(e V)$ & $m_{e}^{*}\left(m_{e}\right)$ \\
\hline $\mathrm{In}_{0.53} \mathrm{Ga}_{0.47} \mathrm{As}$ & 0.75 & 0.36 & 0.041 \\
\hline $\mathrm{InP}$ & 1.42 & 0.11 & 0.079 \\
\hline $\mathrm{GaAs}_{0.5} \mathrm{Sb}_{0.5}$ & 0.81 & 0.75 & 0.040 \\
\hline
\end{tabular}

Table 1. Band parameters for the bulk materials used in the calculation [9].

If one considers an unpolarized beam of incoming conducting electrons and calculates the polarization of the transmitted beam defined by

$$
P(E, \theta)=\frac{T_{+}(E, \theta)-T_{-}(E, \theta)}{T_{+}(E, \theta)+T_{-}(E, \theta)},
$$

one finds, for small number of cells, as shoown in Fig. 2, an increasing maximum polarization for an increasing number of cells in the multiple double-barrier structure. 
We have plotted in Fig. 2 the obtained polarization as a function of electron's energy for transmission across 1,2 and 3 asymmetric double barrier unit cells. The increased polarization with more cells occurs, however, at energies with a corresponding decreased transmission probability, between the infinite superlattice minibans.

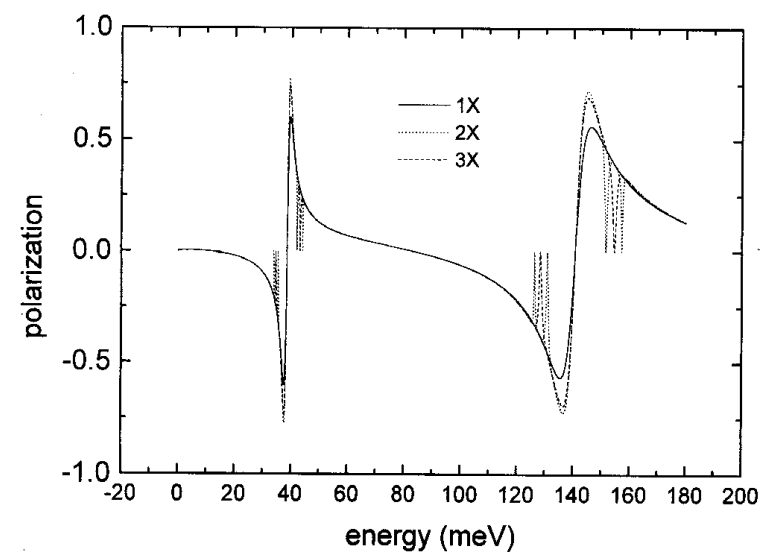

Figure 2. Polarization of the transmitted beam across structures with one $(1 \mathrm{X})$, two $(2 \mathrm{X})$ and three $(3 \mathrm{X})$ repetitions of the asymmetric double-barrier unit cell, with the parameters as in Fig.1.

In conclusion, we have studied the spin-dependent quantum coherent transport of spin polarized electrons along nonmagnetic III-V semiconductor multibarrier nanostructures. We have observed the formation of spin dependent energy minibands with nonzero transmission and an increasing maximum polarization of the transmitted beam with the number of cells, between the minibands, which as mentioned become spin degenare in the superllatice limit. Nevertheles, the interesting situation would be that of minibands strongly dependent on the spin, what is expected to occur in the more general $L_{1} \neq L_{2}$ case, corresponding to superllatices without inversion symmetry. Such study, as well as the analysis of the polarization of the reflected beam, is work in progress and will be published elsewhere.

\section{Acknowledgments}

This work was partially supported by CNPq, CAPES, and FAPESP. We acknowledge helpfull discussions with Prof. G. C. La Rooca.

\section{References}

[1] E. A. de Andrada e Silva and G. C. La Rooca, Phys Rev. B 59, R15583 (1999).

[2] A. Voskoboynikov, S Shin Liu and C. P. Lee, Phys. Rev. B 58, 15397 (1998).

[3] G. Prinz, Phys. Today, 48, 58 (1995).

[4] Spin Eletronics, M. Ziese and M. J. Thornton (Eds), (Springer, Berlin, 2001).

[5] Michael L. Roukes Nature 411, 747 (2001).

[6] Yu A. Bychkov and E. I. Rashba, J. phys. C 17, 6039 (1984).

[7] E. A. de Andrada e Silva, G. C. La Rooca and F. Bassani, Phys Rev B 55, 16293 (1997).

[8] J. Hu, X. G. Xu, J. A. H. Stotz, S. P. Watkins, A. E. Curzon, and M. L. W. Thewalt, N. Matine and C. R. Bolognesi, Appl. Phys. Letters 73, 2799 (1998).

[9] See for instance, Properties of lattice-matched and strained Indium-Gallium-Arsenide, Ed. P. Bhattacharya (Inspec, London, 1993) and Physical properties of III- $V$ semiconductor compounds, Sadao Adachi (John Wiley Sons, New York, 1992). 